\title{
Qualidade sanitária de sementes de coquinho-azedo (Butia capitata) no Norte de Minas Gerais
}

\author{
Health quality of Butia capitata seeds in the north of Minas Gerais, Brazil
}

\author{
Hélida Mara Magalhães ${ }^{\mathrm{I}}$ Hugo César Rodrigues Moreira Catão ${ }^{\mathrm{I}}$ Nilza de Lima Pereira Sales ${ }^{\mathrm{II}^{*}}$ \\ Norma Ferreira de Lima ${ }^{\mathrm{I}}$ Paulo Sérgio Nascimento Lopes ${ }^{\mathrm{II}}$
}

\begin{abstract}
O coquinho-azedo (Butia capitata Mart. Becc.) é uma palmeira nativa dos cerrados brasileiros e seus frutos são fonte de alimento para seres humanos e animais. Os objetivos deste trabalho foram identificar e quantificar os microorganismos nas sementes dessa espécie, utilizando análises "blotter test" $e$ meio Batata-dextrose-ágar (BDA). Amostras foram colhidas em Abóboras, distrito de Montes Claros, Minas Gerais (MG) e, em um esquema fatorial $2 \times 2$, as sementes com e sem endocarpo foram analisadas pelo "blotter test" e BDA, empregando-se o delineamento inteiramente casualisado, com 10 repetições. Essas sementes apresentaram microflora diversificada e a retirada do endocarpo não interferiu na análise microbiológica do coquinho-azedo. Mais espécies de microorganismos foram recuperadas na análise no meio $B D A$, com predominância de fungos Fusarium spp. e Penicillium spp., independentemente da análise. Portanto, a maior parte da microflora identificada nesse trabalho foi fúngica e o melhor teste foi o crescimento em BDA.
\end{abstract}

Palavras-chave: palmeira nativa, frutífera do cerrado, sanidade de sementes.

\section{ABSTRACT}

Coquinho-azedo (Butia capitata Mart. Becc.) is a Brazilian Cerrado native palm tree. Its fruit is used as food to human and animals. This record aim was to identify the microorganisms in the seeds of this species, using the "blotter test" and potato-dextrose-agar (PDA) medium. Samples were collected in Montes Claros, Minas Gerais State. In a factorial scheme $2 \times 2$, seeds with or without endocarp, were analysed by blotter test" and BDA, using a randomly designed with ten replications. These seeds presented a diversified microorganism population and endocarp removing did not interfere in coquinho-azedo microbiological analysis. Greater numbers of microorganism species were recovered in PDA medium, and mostly were Fusarium spp. and Penicillium spp. This study concluded that predominantly B. capitata microorganism population was fungus and PDA was better medium.

Key words: native palm, fruitful tree, health analysis.

O coquinho-azedo (Butia capitata Mart. Becc.) é uma palmeira nativa dos cerrados brasileiros, encontrada principalmente nos Estados da Bahia, de Goiás e de Minas Gerais. Ela destaca-se pelo intenso uso na alimentação regional. Seus frutos são utilizados para o consumo in natura e para o preparo de sucos, sorvetes e picolés (MARTINS, 2003). O coquinho azedo desempenha importante papel socioeconômico junto às comunidades carentes do Norte de Minas em função, principalmente, do extrativismo de seus frutos. Atualmente, com a expansão das fronteiras agrícolas e com o extrativismo intenso, esta espécie encontra-se ameaçada.

A propagação do coquinho-azedo é feita exclusivamente via seminífera, tornando imprescindível à obtenção de sementes de boa qualidade fisiológica e sanitária. No entantos muitos microorganismos são veiculados pelas sementes e são causadores de danos em pré e pós-emergência. Estes ocasionam plântulas anormais, podridões radiculares, infecções no sistema vascular, tombamento, manchas necróticas em folhas,

ICurso de Agronomia, Núcleo de Ciências Agrárias (NCA) Universidade Federal de Minas Gerais (UFMG), Belo Horizonte, MG, Brasil.

"Setor de Fitotecnia, UFMG, 39404-006, Belo Horizonte, MG, Brasil. E-mail: nsales@nca.ufmg.br. *Autor para correspondência. 
caules e frutos, sendo que outros danos podem ser provocados na própria semente, tais como perda no potencial germinativo, abortos e estromatizações (FERREIRA, 1989; SALES, 1994; CHEROBIN et al., 2004; FAIAD et al., 2004).

Pouco se sabe sobre a associação de microorganismos com as sementes de espécies nativas do cerrado, principalmente, quando se trata do coquinho-azedo. Além disso suas formas de acesso não são conhecidas, muito menos os danos que os mesmos provocam nas sementes, na sua emergência ou no estabelecimento de plantas no campo.

Diante do exposto, os objetivos deste estudo foram identificar e quantificar os microorganismos presentes nas sementes de coquinhoazedo com e sem endocarpo.

Frutos maduros de coquinho-azedo foram colhidos na região de Montes Claros, Minas Gerais (MG), em janeiro de 2007 e o presente experimento foi realizado de 30/03 a 14/04 do mesmo ano. As sementes foram beneficiadas e acondicionadas em sacos de papel por dois meses, em temperatura ambiente.

As sementes foram analisadas utilizando os métodos do papel de filtro (blotter test) e meio BDA (batata-dextrose-ágar), de acordo com MACHADO (2000), com algumas modificaçãoes. Sementes com e sem endocarpo foram desinfetadas em hipoclorito de sódio $2 \%$ por três minutos. Em seguida, elas foram lavadas por três vezes com água destilada esterilizada e secas na capela de fluxo laminar, antes da semeadura em caixas gerbox com papel germinativo ou mata borrão, ou em placas de Petri com meio BDA. Os endocarpos das sementes foram retirados manualmente. Todos os procedimentos foram, realizados sob condições assépticas em câmara de fluxo laminar.

As sementes foram acondicionadas em incubadora por 15 dias, sob fotoperíodo de 12 horas de luz e temperatura de $25 \pm 2^{\circ} \mathrm{C}$, e os microorganismos foram identificados utilizando microscópio estereoscópico (20x) e, quando necessário, foram preparadas lâminas microscópicas com as estruturas dos fungos para a visualização ao microscópio composto.

O delineamento inteiramente casualizado foi empregado com 10 repetições de 10 sementes por parcela, em esquema fatorial 2 × 2 (sementes com e sem endocarpo, submetidas aos métodos blotter test e BDA). Os dados de porcentagem de ocorrência dos microorganismos foram submetidos à análise de variância e as médias foram comparadas pelo teste de Tukey a $5 \%$ de probabilidade de erro. Os dados foram transformados em $\sqrt{0,5+x}$ antes da análise.

$\mathrm{Na}$ avaliação dos microorganismos presentes nas sementes de coquinho-azedo, independente do tratamento da semente e do método de análise, foram detectados oito fungos e um actinomiceto (Tabela 1). A interação tratamento da semente e método de análise não foi significativa, assim como o efeito isolado do tratamento da semente (com e sem endocarpo).

$\mathrm{O}$ método de análise foi altamente significativo (Tabela 2), distinguindo o meio BDA como superior ao método do papel de filtro para o levantamento da microflora das sementes do coquinhoazedo. O meio BDA é mais rico em nutrientes, ao contrário do blotter, e isso provavelmente favoreceu uma maior recuperação de fungos.

Resultados semelhantes foram obtidos por ARAÚJO et al. (2004), analisando sementes de amendoim (Arachis hypogaea L.) e utilizando diversos procedimentos de incubação. Os pesquisadores concluíram que o meio BDA com restrição hídrica propiciou maior recuperação de fungos como Cladosporium spp., Penicillium spp., Aspergillus flavus, A. niger, A. glaucus.

Independente do tratamento da semente (com e sem endocarpo) e do método de análise (BDA e blotter test), no geral, os fungos Fusarium spp. (72,3\%) e Penicillium spp. (32,5\%) foram os que mais ocorreram.

Em sementes de fava d'anta (Dimorphandra mollis), ARAÚJO et al. (2007) identificaram a presença de 12 fungos, independente do método de colheita (frutos do solo ou frutos da árvore). Destes, quatro foram também identificados nas sementes de coquinhoazedo, sendo eles: Aspergillus spp., Cladosporium spp., Fusarium spp., e Penicillium spp. Estes são comumente encontrados nas sementes de essências florestais que, normalmente, se contaminam quando os frutos caem no solo e se propagam durante o beneficiamento e o armazenamento dos frutos e, de acordo com as condições de armazenamento, podem comprometer a qualidade das sementes, reduzindo o seu poder germinativo ou ocasionando a morte de embriões.

Algumas espécies de Fusarium sp. têm sido relatadas causando tombamento em pré- ou pósemergência de plântulas de espécies florestais, sendo problema comum em sementes destas espécies (FERREIRA, 1989; SALES, 1994; CHEROBIN et al., 2004).

Em sementes de caupi, Aspergillus spp. é comumente citado como patógeno. Nas sementes, o fungo permanece por muito tempo e, normalmente, não causa problemas em condições de campo, entretanto, durante o armazenamento, produzem toxinas que afetam 
Tabela 1 - Ocorrência de microrganismos associados às sementes de Butia capitata Mart. Becc., com e sem endocarpo, submetidas ao método de Blotter e meio Batata-dextrose-ágar. Montes Claros-MG.

\begin{tabular}{|c|c|c|c|c|c|}
\hline \multirow[t]{2}{*}{ Microrganismos } & \multicolumn{2}{|c|}{ Semente com endocarpo } & \multicolumn{2}{|c|}{ Semente sem endocarpo } & \multirow{2}{*}{ Média } \\
\hline & BDA* & Blotter & BDA & Blotter & \\
\hline Actinomiceto & 20 & 0 & 20 & 0 & 10,0 \\
\hline Aspergillus flavus & 5 & 0 & 24 & 4 & 8,3 \\
\hline Aspergillus niger & 33 & 7 & 2 & 0 & 10,5 \\
\hline Cladosporium sp. & 0 & 0 & 6 & 0 & 1,5 \\
\hline Epicoccum sp. & 1 & 0 & 1 & 0 & 0,5 \\
\hline Fusarium sp. & 77 & 61 & 80 & 71 & 72,3 \\
\hline Mucor sp. & 17 & 0 & 8 & 0 & 6,3 \\
\hline Penicillium sp. & 60 & 37 & 23 & 10 & 32,5 \\
\hline Trichoderma sp. & 0 & 0 & 22 & 0 & 5,5 \\
\hline
\end{tabular}

*BDA = Batata-dextrose-ágar.

e causam deterioração das sementes, além de afetarem sua qualidade (AGARWAL \& SINCLAIR, 1987).

Quanto ao fungo Cladosporium sp., este foi relatado em freqüentes associações com sementes de 58 espécies florestais nativas do cerrado brasileiro, causando descoloração das sementes, redução da taxa de germinação, queda do vigor das plântulas, escurecimento das sementes, provocando deterioração do endosperma, necrose nas raízes e morte de plântulas em viveiro de modo geral (FAIAD et al., 2004).

Em relação aos fungos Mucor sp.,

Trichoderma sp. e Epicoccum sp., não há relatos como patogênicos a espécies florestais e frutíferas. Trichoderma sp. é considerado fungo saprófita, utilizado em estudos como agente de controle biológico. Epiccocum sp. foi encontrado associado às sementes de ipê amarelo e de ipê-roxo. Entretanto,

Tabela 2 - Número de microrganismos identificados nas sementes de Butia capitata, analisadas por meio os métodos Blotter e Batata-dextrose-ágar. Montes Claros-MG.

\begin{tabular}{llll}
\hline Tipo de semente & \multicolumn{3}{c}{------Método de análise---------- } \\
& BDA* & Blotter & Média \\
\hline & & & \\
Com endocarpo & 4,80 & 2,00 & $3,4 \mathrm{a}$ \\
Sem endocarpo & 5,40 & 2,10 & $3,75 \mathrm{a}$ \\
Média & $5,05 \mathrm{~A}$ & $2,05 \mathrm{~B}$ & \\
CV(\%) & & & 12,58 \\
\hline
\end{tabular}

*BDA = Batata-dextrose-ágar.

Médias não seguidas da mesma letra maiúscula na linha e minúscula na coluna diferem estatisticamente entre si a $5 \%$ de probabilidade de erro pelo teste Tukey. não foi constatado efeito negativo na germinação dessas espécies, bem como presença de plântulas doentes (BOTELHO, 2006).

Quanto ao actinomiceto avaliado, não foram encontrados relatos deste microrganismo em sementes de espécies frutíferas e florestais, porém, SILVA(2004), testando 103 isolados de actinomicetos sobre a germinação de sementes de pepino (Cucumis salivus L) e sorgo (Sorghum bicolor), verificou que 18,5\% dos isolados apresentaram efeito fito-inibitório nas plantasteste.

Mais espécies de microorganismos foram recuperadas na análise no meio $\mathrm{BDA}$, sendo que os fungos que predominaram foram Fusarium spp. e Penicillium spp., independentemente da análise.

\section{REFERÊNCIAS}

ARAÚJO, A.E. da. et al. Avaliação de metodologia para detecção de fungos em sementes de amendoim. Revista Brasileira Sementes, Pelotas, v.26,n.2, p.45-54, 2004.

ARAÚJO, A.V. et al. Avaliação sanitária e fisiológica de sementes de Fava D'anta (Dimorphandra mollis Benth.) obtidas de frutos coletados no solo e na planta. In: CONGRESSO BRASILEIRO DE OLERICULTURA; SIMPÓSIO BRASILEIRO SOBRE CURCUBITÁCEAS, 47., 2006, Porto Seguro, BA. Anais... Porto Seguro: Horticultura Brasileira, 2007. V.25, n.1. p.280 p.33.

AGARWAL, V.K.; SINCLAIR, J.B. Principles of seed pathology. Boca Raton, Flórida: CRC, 1987. 2v. 176p.

BOTELHO, L.S. Fungos associados às sementes de ipêamarelo (Tabebuia serratifolia), ipê-roxo (Tabebuia impetiginosa), aroeira-pimenteira (Schinus terebinthifolius) e aroeira-salsa (Schinus molle): incidência, efeitos na germinação, transmissão para plântulas e controle. 2006. 114f. Dissertação (Mestrado em agronomia) - Curso de 
Pós-graduação em agronomia, Escola Superior Luiz de Queiroz, Universidade de São Paulo, Piracicaba.

CHEROBIN, E.A. et al. Fungos associados às sementes de guapuruvú (Schizolobiump parahyba) provenientes dos estados do Rio Grande do Sul, Santa Catarina e Paraná. In: SIMPÓSIO BRASILEIRO DE PATOLOGIA DE SEMENTES, 8., 2004, João Pessoa, PB. Resumos... João Pessoa: Universidade Federal da Paraiba, 2004. 260p. p.162.

FAIAD, M.G.R. et al. Patologia de espécies florestais do cerrado. In: SIMPÓSIO BRASILEIRO DE PATOLOGIA DE SEMENTES, 8., 2004, João Pessoa. Palestras e Resumos... João Pessoa, 2004. p.71.

FERREIRA, F.A. Patologia florestal: principais doenças florestais no Brasil. Viçosa: Sociedade de investigações Florestais, 1989. 570p.
MACHADO, J da C. Tratamento de sementes no controle de doenças. Lavras: PS/UFLA/FAEPE, 2000. 138p.

MARTINS, E.R. Projeto conservação de recursos genéticos de espécies frutíferas nativas do Norte Mineiro: coleta, ecogeografia e etnobotânica. Montes Claros: UFMG, 2003. 76p. (Relatório Institucional).

SALES, N.L.P. Efeito da população fúngica sobre a germinação das sementes e o desenvolvimento inicial de plântulas de ipêamarelo (Tabebuia serratifolia) e barbatimão (Stryphnodendron adstringes). Ciência e Prática, Lavras, v.18, n.1, p.8389,1994 .

SILVA, F.A.M. Seleção de microrganismos com potencial de produção de compostos alelopáticos para o controle de plantas daninhas. 2004. 61f. Dissertação (Mestrado em Agronomia) - Curso de Pós- graduação em Agronomia, Escola Superior de Agricultura Luiz de Queiroz, Universidade de São Paulo, Piracicaba. 